\title{
Technical note: Quantifying and characterizing behavior in dairy calves using the IceTag automatic recording device
}

\author{
P. Trénel, ${ }^{* 1}$ M. B. Jensen, $†$ E. L. Decker,† and F. Skjøth* \\ ${ }^{*}$ AgroTech A/S, Institute for Agro Technology and Food Innovation, Udkærsvej 15, DK 8200 Aarhus, Denmark \\ †University of Aarhus, Faculty of Agricultural Sciences, Department of Animal Health, Welfare and Nutrition, Research Centre Foulum, \\ Blichers Allé, Postbox 50, DK 8830 Tjele, Denmark
}

\section{ABSTRACT}

The objectives of the current study were 1) to validate the IceTag (http://www.icerobotics.com) automatic recording device for measuring lying, standing, and moving behavior in dairy calves, and 2) to improve the information yield from this device by applying a filtering procedure allowing for the detection of lying versus upright. The IceTag device provides measures of intensity (I) of lying, standing, and activity measured as percent lying, percent standing, and percent active, but does not directly measure lying, standing, and moving behavior because body movements occurring while lying (e.g., shifts in lying position) and while upright (e.g., grooming) are recorded as activity. Therefore, the following 3-step procedure was applied. First, thresholds for I were determined by choosing the cutoff that maximized the sum of sensitivity (Se) and specificity (Sp). Second, a lying period criterion (LPC) was established empirically, and IceTag data were filtered according to the LPC, providing information on the posture of the animal as lying versus being upright. Third, a new threshold of I was estimated for moving activity conditional on the animal being upright. IceTag recordings from 9 calves were compared with video recordings during a 12 -h period and analyzed using $2 \times 2$ contingency tables. Data from the first 4 calves were used to determine an LPC, whereas the remaining 5 calves served for validation of the procedure. An optimal LPC was found by modeling the deviance between IceTag and video recordings as a function of the LPC and choosing the LPC threshold that minimized the deviance. The IceTag device was found to accurately measure the high-prevalence behaviors (lying and standing; Se+Sp >1.90) and less accurately measure the low-prevalence behavior (moving; Se+Sp $=1.39$ ). Application of the 3 -step procedure using an

Received January 16, 2009

Accepted March 4, 2009

${ }^{1}$ Corresponding author: pht@agrotech.dk optimal LPC estimate of $24.8 \mathrm{~s}$ resulted in an improved description of calf behavior, yielding a valid representation of the number and duration of lying and upright periods $(\mathrm{Se}+\mathrm{Sp}=2.00)$ within a precision of 0 to $49 \mathrm{~s}$ (95\% confidence interval). In group-housed dairy calves, valid measures of the number and duration of lying and upright periods may be obtained from the IceTag device when applying the presented filtering procedure to the data. Measures regarding locomotion, on the other hand, should be used with caution.

Key words: activity, lying, standing, validation

Behavioral monitoring may help improve animal welfare and productivity in farm animals, because behavior provides relevant information on food and water intake (Chapinal et al., 2007) as well as health state (Urton et al., 2005; Huzzey et al., 2007). Until recently, animal behavior has been quantifiable only by means of direct observation or video monitoring, both of which are costly in terms of labor and time (Müller and Schrader, 2003).

A variety of automatic recording techniques for the measurement of behavior have been developed (Müller and Schrader, 2003; Munksgaard et al., 2006). Validation studies using video observations as a control have reported high levels of correspondence between video recording and automatic devices when considering the total duration of behavioral activities (Müller and Schrader, 2003; Munksgaard et al., 2006). However, the daily duration of a behavioral activity may not be a satisfactory descriptor of the actual behavior because of the typical nested structure of many behavioral activities (Magnusson, 2000). For instance, in cattle, lying behavior may occur in bouts of several discrete lying periods, with short intervening phases of standing. Often, records of the number of postural changes from lying to standing are of prime interest (e.g., Haley et al., 2001). A discrete lying period may in turn contain minor movements; for example, shifts in lying position or grooming while lying. Similarly, a standing animal may make sporadic movements; for example, arching the back while defecating, without "leaving the spot." 
Moving periods may contain instances of short stops or sudden direction changes without having ended the sequence of body movements. Recording of these intraperiod behavioral changes by the automatic recording device could blur important patterns in major behavioral sequences. Hence, data modeling may be required before relevant information on animal behavior can be extracted from automated recording devices.

Lying, standing, and moving behavior may preferably be described as realizations in a 2-dimensional continuous space defined by a lying/upright axis and an active/inactive axis, with lying behavior corresponding to a combination of lying and displaying low to high activity, standing behavior corresponding to being upright and displaying low activity, and moving behavior corresponding to being upright and displaying high activity. Obviously, transitions between states occur smoothly. Nevertheless, transitions between lying and upright may be more sharply separated than transitions between moving and standing because of physical constraints on intermediate states. Hence, distinction between lying versus upright may be a natural starting point for accessing the nested structure of animal behavior.

The aims of the current study were 1) to evaluate the validity of a commercially available, accelerometerbased automatic recording device for measuring lying, standing, and moving behavior in dairy calves kept in a loose housing system; and 2) to improve the information yield from this device by sequentially making use of recorded lying intensity information, a lying period criterion (LPC) to define the animal as lying or upright, and moving intensity information to determine the animal's activity state while being upright.

The IceTag automatic recording device (IceRobotics, Edinburgh, UK; http://www.icerobotics.com; dimensions $(\mathrm{mm}): 95.0$ high $\times 82.3$ wide $\times 31.5$ deep; weight: $130 \mathrm{~g})$ provides for each recorded second a measure of intensity (I) of lying (percent lying), standing (percent standing), and moving (percent active) based on 8 recordings per second. For instance, 8 recordings of lying within 1 s results in a measure of $100 \%$ lying for that second. The IceTag device was attached by means of a strap to the right hind leg above the fetlock of 9 (3 male and 6 female) Holstein calves (2 mo of age). All 9 calves were used to validate the IceTag device (data set 1 ). An LPC estimate was obtained based on the 4 of the 9 calves (data set 2 ), with the remaining 5 calves (data set 3) used to evaluate the application of the LPC. Raw data were transferred from the IceTag device to a computer and processed using the IceTagAnalyser software; data (hereafter termed IceTag data) on the average percent lying, standing, and activity were ob- tained on a 1-s basis. The 9 calves were selected among 24 calves housed in groups of 4 (each group containing 2 male and 2 female calves) in straw-bedded pens (2.49 $\mathrm{m} \times 4.15 \mathrm{~m})$. At least one calf from each group was selected. Calves were offered concentrate (18\% CP; Grøn Kalv Valset, DLG, Copenhagen, Denmark), hay, and water ad libitum. Concentrate and hay were provided at $0900 \mathrm{~h}$, and pens were bedded with fresh straw. All calves were individually marked by bleaching of the fur. Data were recorded by the IceTag and the behavior was recorded via video in a 12-h period from 0900 to 2100 $\mathrm{h}$. From the video recordings, the behavior of individual calves was recorded continuously by 2 trained observers. The start and end time of "lying" (lying on sternum or on side), "standing" (body upright and supported by at least 3 legs), "walking" (at least 3 legs are moved forward in sequence), and "running" (trotting or galloping) were recorded with 1-s accuracy. "Walking" and "running" were subsequently combined to "moving" because there were too few recordings of running.

The following 3-step procedure was applied. First, correspondence at the level of the recorded time unit (1 s) between the IceTag (test data) and video data (standard) was analyzed by $2 \times 2$ contingency tables (true positives, false negatives, false positives, and true negatives). Sensitivity (Se; true positives out of sum of true positives and false negatives) and specificity ( $\mathbf{S p}$; true negatives out of sum of true negatives and false positives) were calculated for different cutoff levels for IceTag-recorded intensities (i.e., lying intensity, LI; standing intensity, SI; and moving intensity, MI) following Dohoo et al. (2003) and assuming a gold standard (video recordings). The analyzed cutoff levels were $12.5,25.0,37.5,50.0,62.5,75.0,87.5$, and $100.0 \%$, corresponding to the 8 possible outcomes of intensity values I provided by the IceTag device (based on 8 recordings per second). Optimal cutoff levels for LI, SI, and MI were found for each calf as those that maximized the sum of Se and Sp. Second, an optimal LPC was determined in the following way: 1) IceTag nonlying periods $\left(t_{T}\right)$ were determined as intervals between IceTag records with $\mathrm{LI} \geq \mathrm{LI}_{\text {optimal }}$; 2) for each cutoff (c) of $t_{\mathrm{T}}$, the difference between $t_{\mathrm{T}}$ and the video nonlying intervals $\left(t_{\mathrm{V}}\right)$ was determined $\left(d_{\mathrm{c}}=\mid \Sigma\left(t_{\mathrm{T}}\right)-\right.$ $\left.\Sigma\left(t_{\mathrm{V}}\right) \mid\right)$; 3) then, $\log \left(d_{\mathrm{c}}\right)$ was regressed against $\log (\mathrm{c})$ using generalized additive models [Wood, 2006; $\log \left(d_{\mathrm{c}}\right)$ $\sim \mathrm{s}(\log (\mathrm{c}))]$. A generalized additive model is a generalized linear model with a linear predictor involving a sum of smooth functions of covariates and, hence, is especially well suited for modeling bumped distributions (Wood, 2006). The $\mathrm{LPC}_{\text {optimal }}$ was found as the cutoff level where the prediction of $\log \left(d_{\mathrm{c}}\right)$ was minimized. Calculations were done on the pooled data set 1 . In the 
Table 1. Test evaluation statistics for IceTag (http://www.icerobotics.com) raw data (IceTag) and processed IceTag (IceTag ${ }_{\text {LPC }}$ ) according to the 3-step filtering procedure presented in the current study

\begin{tabular}{|c|c|c|c|c|}
\hline Item & Sensitivity (Se) & Specificity (Sp) & $\mathrm{Se}+\mathrm{Sp}$ & $\mathrm{n}$ \\
\hline \multicolumn{5}{|l|}{ IceTag $^{1}$} \\
\hline \multicolumn{5}{|l|}{ Lying } \\
\hline Mean $\pm \mathrm{SE}^{2,3}$ & $0.996 \pm<0.001$ & $0.980 \pm<0.001$ & $1.975 \pm<0.001$ & 63 \\
\hline $\mathrm{I}_{\text {opt }}>50 \%^{3}$ & $0.996 \pm<0.001$ & $0.982 \pm<0.001$ & $1.978 \pm<0.001$ & 9 \\
\hline \multicolumn{5}{|l|}{ Standing } \\
\hline Mean $\pm \mathrm{SE}^{2}$ & $0.921 \pm 0.001$ & $0.977 \pm<0.001$ & $1.900 \pm 0.001$ & 72 \\
\hline $\mathrm{I}_{\mathrm{opt}} \geq 37.5 \%^{4}$ & $0.919 \pm 0.005$ & $0.984 \pm<0.001$ & $1.903 \pm 0.005$ & 9 \\
\hline \multicolumn{5}{|l|}{ Moving } \\
\hline Mean $\pm \mathrm{SE}^{2,5}$ & $0.154 \pm 0.001$ & $0.979 \pm<0.001$ & $1.113 \pm 0.001$ & 64 \\
\hline $\mathrm{I}_{\text {opt }} \geq 50 \%$ & $0.160 \pm 0.009$ & $0.979 \pm 0.001$ & $1.139 \pm 0.007$ & 8 \\
\hline \multicolumn{5}{|l|}{ IceTag $_{\text {LPC }}{ }^{6}$} \\
\hline \multicolumn{5}{|l|}{ Lying } \\
\hline Mean $\pm \mathrm{SE}^{4}$ & $0.994 \pm<0.001$ & $0.990 \pm 0.001$ & $1.985 \pm 0.002$ & 5 \\
\hline \multicolumn{5}{|l|}{ Moving/upright } \\
\hline Mean $\pm \mathrm{SE}^{2,5}$ & $0.221 \pm 0.002$ & $0.971 \pm<0.001$ & $1.192 \pm 0.001$ & 32 \\
\hline $\mathrm{I}_{\mathrm{opt}} \geq 50 \%$ & $0.229 \pm 0.015$ & $0.971 \pm 0.002$ & $1.200 \pm 0.013$ & 4 \\
\hline Lying-down periods, ${ }^{4} \mathrm{n}$ & $1.000 \pm 0.000$ & $1.000 \pm 0.000$ & $2.000 \pm 0.000$ & 5 \\
\hline \multicolumn{5}{|c|}{${ }^{1}$ Data set 1 , including 9 calves. } \\
\hline \multirow{3}{*}{\multicolumn{5}{|c|}{$\begin{array}{l}{ }^{2} \text { Across calves and intensity thresholds I, with I }>0 \text {; that is, } 12.5,25,37.5,50,62.5,75,87.5 \text {, and } 100 \% \text {. } \\
{ }^{3} \mathrm{I}_{\mathrm{opt}}=\text { intensity threshold that maximized Se }+ \text { Sp; no recordings of lying intensity of } 12.5 \% \text { were recorded b } \\
\text { the IceTag in any of the } 9 \text { calves, resulting in a total of } 7 \text { intensity thresholds for lying. }\end{array}$}} \\
\hline & & & & \\
\hline${ }^{4}$ Across calves. & & & & \\
\hline \multicolumn{5}{|c|}{$\begin{array}{l}{ }^{5} \mathrm{~A} \text { single calf was not recorded as walking according to video observation and was therefore excluded befor } \\
\text { calculations. }\end{array}$} \\
\hline
\end{tabular}

final step, $\mathrm{MI}_{\text {optimal }}$ was recalculated for moving activity recordings conditional on the animal being upright, and moving activity was estimated as recordings of $\mathrm{MI} \geq$ $\mathrm{MI}_{\text {optimal }}$ | upright.

Video observation detected lying as the most dominant behavioral pattern in all 9 calves (prevalence $=$ $0.70 \pm 0.02$ ). Standing was intermediate (prevalence $=0.28 \pm 0.02)$, whereas moving had a low prevalence (prevalence $=0.02 \pm 0.01$ ). A total of 105 lying periods were observed in the video data, with 9 to 19 lying periods per calf. Across all calves, the shortest and longest observed lying periods varied in duration between $67 \mathrm{~s}$ and $26.7 \mathrm{~min}$, and between $84.0 \mathrm{~min}$ and $153.3 \mathrm{~min}$, respectively. Lying periods displayed a slightly bimodal distribution, with modes around 150 $\mathrm{s}$ and $58.3 \mathrm{~min}$ (data not shown). Upright (i.e., nonlying) periods ranged between $78 \mathrm{~s}$ and $56.7 \mathrm{~min}$, with standing periods varying between $1 \mathrm{~s}$ and $38.3 \mathrm{~min}$, and moving periods varying between $1 \mathrm{~s}$ and $7.4 \mathrm{~min}$. The distributions of standing and moving were both strongly right-skewed $\left(\right.$ median $_{\text {standing }}=64 \mathrm{~s} ;$ median $_{\text {moving }}$ $=6 \mathrm{~s}$ ), displaying high frequencies of periods $<1 \mathrm{~min}$ and $<10 \mathrm{~s}$, respectively, indicating that transitions between standing and moving occurred at high frequency. Transitions between behavioral states appeared to be ordered and bidirectional following a lying $\leftrightarrow$ standing $\leftrightarrow$ moving path, where short instances of standing intervals intervening lying and moving periods (one to a few seconds) corresponded to "getting up" and "lying down" behavior.

The IceTag recording device provided data of high Se and Sp at the level of the recorded time unit (s) for the intermediate to high prevalence behavioral patterns (lying and standing; Table 1; data set 1). This was true for all nonzero cutoff levels for recorded activity intensities, with little difference observed among them (Table 1). Optimal intensity thresholds for lying and standing were found at $\mathrm{LI}_{\text {optimal }} \geq 50 \%$ and $\mathrm{SI}_{\text {optimal }} \geq 37.5 \%$. Using these cutoff levels, the IceTag displays predictive values (probability of correct positive, $P V+$, and negative, $P V$-, respectively; Dohoo et al., 2003) for lying and standing behavior close to $1.00\left(P V+_{\text {lying }}=0.994 \pm\right.$ $<0.001, P V-_{\text {lying }}=0.987 \pm 0.001 ; P V+_{\text {standing }}=0.949$ $\left.\pm 0.002, P V-_{\text {standing }}=0.973 \pm 0.002\right)$. In contrast, moving was poorly captured by the IceTag device (Table $1)$. At the optimal intensity threshold $\left(\mathrm{MI}_{\text {optimal }} \geq 50 \%\right)$, recordings of moving activity displayed low levels of Se and greater among-calf variability compared with lying and standing behavior (Table 1). The probability of an IceTag recording of moving corresponding to true moving behavior was worse than $50 \%\left(P V+_{\text {moving }}=0.130 \pm\right.$ $0.003)$, stressing that moving activity was consistently overestimated by the IceTag device $(P V-/ P V+=7.57$; Figure 1).

At the optimal LI cutoff of $50 \%$, IceTag-recorded nonlying intervals varied in length from 1 to $2,628 \mathrm{~s}$ 


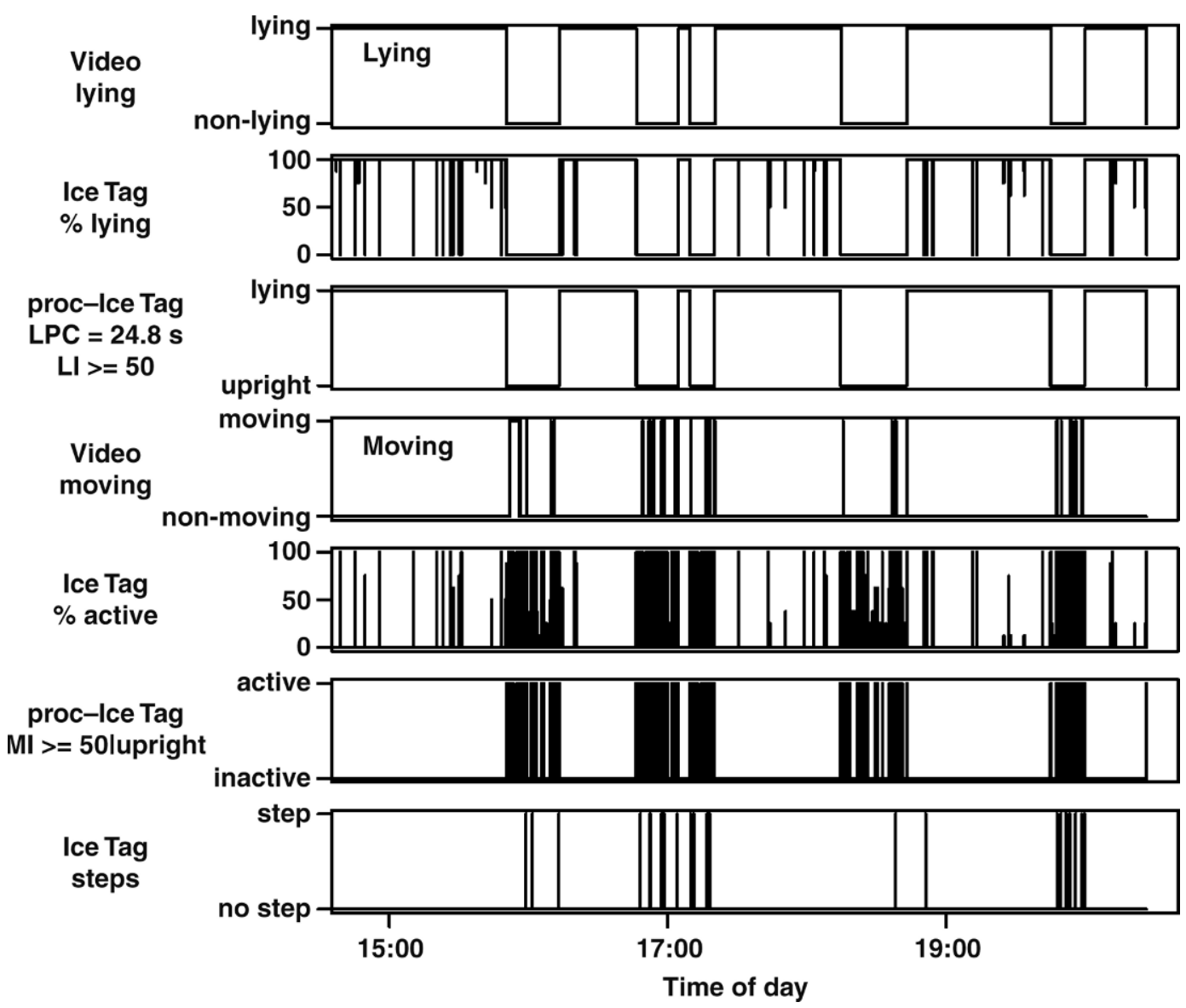

Figure 1. Lying and moving patterns of one subject during part of the observation period as recorded by video, the IceTag (http://www. icerobotics.com), and by processed IceTag (proc-IceTag). IceTag-recorded step sequences of duration $>1 \mathrm{~s}$ are also shown. LPC $=1$ lying period criterion; LI = lying intensity; $\mathrm{MI}=$ moving intensity.

( $\sim 47 \mathrm{~min})$, with a high frequency $(63 \%)$ of 1-s nonlying recordings (data set 2 ). This resulted in an optimal LPC of 24.8 s. No overlap was found in the distributions of IceTag-detected nonlying intervals within video-detected lying periods and between video-detected lying periods (data not shown). Application of the LPC (data set 3) resulted in an improved description of calf behavior (Table 1, Figure 1). All lying and upright periods could be validly recovered (Table 1, Figure 1). Precision of the duration of recovered lying periods was found to be within seconds (geometric mean $=6 \mathrm{~s}$, $95 \%$ confidence interval $=[0,49] \mathrm{s})$. Application of the 3 -step procedure resulted in a slight improvement in the sensitivity of the IceTag to moving activity (Table 1 ). However, moving activity remained poorly described and consistently overestimated (Table 1; Figure 1).

The current study demonstrated that the IceTag automated recording device could accurately estimate the high-prevalence activities (lying and standing) in calves kept in a loose housing system. The IceTag has previously been validated for use in dairy cattle by
Munksgaard et al. (2006), who found high correlations $(>0.84)$ between video and IceTag recordings. Using an arbitrary lying period criterion of $5 \mathrm{~s}$ (L. Munksgaard, University of Aarhus, Denmark; personal communication), duration and frequency of bouts of moving and standing could be estimated with high precision in dairy cows in a runway (Munksgaard et al., 2006). In the current study, the LPC was empirically estimated to $24.8 \mathrm{~s}$. Calf behavior is known to vary with factors such as age, sex, feeding, and housing conditions; for instance, time spent lying is affected by age (Jensen et al., 1998) as well as milk allowance (De Paula Vieira et al., 2008). Because there was no overlap of the distributions of IceTag-detected nonlying intervals within and between video-detected lying periods observed in the current study, some robustness of our LPC estimate is suggested. However, future studies using data from automatic behavior recording devices and behavioral observations for different age groups under different housing conditions would be needed for an assessment of LPC under these conditions. 
Application of the LPC in the current study improved the information yield from the IceTag considerably by providing valid information on the number and durations of lying and upright periods. Moving behavior, on the other hand, was not well represented and was consistently overestimated by the IceTag because of a high frequency of movements occurring nested within lying and standing behavior. This stresses that inference on moving behavior based on accelerometer-based automatic animal behavior recording devices such as the IceTag may be limited and should be used with caution. Moving included walking and running and it may be desirable for a recording device to be able to distinguish the two, especially in calves where running is a frequent part of play behavior (Jensen et al., 1998). Besides intensity of lying, standing, and moving, the IceTagAnalyser-processed IceTag data include, for each second, the number of steps taken. A combination of information from recorded moving activity and steps may be necessary to achieve a valid representation of locomotor behavior in calves (Figure 1).

\section{ACKNOWLEDGMENTS}

This study was funded by The Danish Ministry of Food, Agriculture and Fisheries (Copenhagen, Denmark) and The Danish Cattle Federation (Aarhus, Denmark). The authors thank John Misa Obidah, Betty Skou, and Carsten K. Christensen (University of Aarhus, Denmark) for assisting the data collection, and Mette S. Herskin and Lene Munksgaard (Univer- sity of Aarhus, Denmark) for valuable comments on the manuscript.

\section{REFERENCES}

Chapinal, N., D. M. Veira, D. M. Weary, and M. A. G. von Keyserlingk. 2007. Technical note: Validation of a system for monitoring individual feeding and drinking behavior and intake in grouphoused cattle. J. Dairy Sci. 90:5732-5736.

De Paula Vieira, A., V. Guesdon, A. M. Passillé, M. A. G. von Keyserlingk, and D. M. Weary. 2008. Behavioural indicators of hunger in dairy calves. Appl. Anim. Behav. Sci. 109:180-189.

Dohoo, I., W. Martin, and H. Stryhn. 2003. Veterinary Epidemiologic Research. AVC Inc., Charlottetown, Canada.

Haley, D. B., A. M. de Passillé, and J. Rushen. 2001. Assessing cow comfort: Effects of two floor types and two tie stall designs on the behaviour of lactating dairy cows. Appl. Anim. Behav. Sci. $71: 105-117$

Huzzey, J. M., D. M. Veira, D. M. Weary, and M. A. G. von Keyserlingk. 2007. Prepartum behavior and dry matter intake identify dairy cows at risk for metritis. J. Dairy Sci. 90:3220-3233.

Jensen, M. B., K. S. Vestergaard, and C. C. Krohn. 1998. Play behaviour in domestic calves kept in pens: The effect of social contact and space allowance. Appl. Anim. Behav. Sci. 56:97-108.

Magnusson, M. S. 2000. Discovering hidden time patterns in behavior: T-patterns and their detection. Behav. Res. Methods Instrum. Comput. 32:93-110.

Müller, R., and L. Schrader. 2003. A new method to measure behavioural activity levels in dairy cows. Appl. Anim. Behav. Sci. $83: 247-258$.

Munksgaard, L., C. G. Reenen, and R. Boyce. 2006. Automatic monitoring of lying, standing and walking behavior in dairy cattle. J. Anim. Sci. 84(Suppl.):304.

Urton, G., M. A. G. von Keyserlingk, and D. M. Weary. 2005. Feeding behavior identifies dairy cows at risk for metritis. J. Dairy Sci. 88:2843-2849.

Wood, S. N. 2006. Generalized Additive Models: An Introduction with R. Chapman and Hall/CRC, New York, NY. 